INTERnational JOURNAL OF MULtidisciplinaRy Research AND ANALysis

ISSN(print): 2643-9840, ISSN(online): 2643-9875

Volume 05 Issue 01 January 2022

DOI: 10.47191/ijmra/v5-i1-07, Impact Factor: 6.072

Page No.- 53-62

\title{
A Comparative Study on Webpage Browsing Performance between Proprietary and Open Source Operating Systems on Wireless Networks
}

\author{
Sharra Mae B. Fernandez ${ }^{1}$, Bella Gertrude B. Alpasan ${ }^{2}$, Mary Jane V. Esimos ${ }^{3}$, Audrey Karyl P. \\ Maligang ${ }^{4}$, Sheila Mae S. Pagayonan ${ }^{5}$, April Rose A. Zaragosa ${ }^{6}$ \\ 1,2,3,4,5,6 Northern Iloilo Polytechnic State College, Estancia, lloilo
}

ABSTRACT: This experimental research study determined and compared the webpage browsing performance of proprietary and open source operating systems on wireless networks. It was intended to reveal the significant differences in the webpage browsing performance between proprietary and open source operating systems on wireless networks when classified as to hardware specifications and type's web content. The researchers used the JavaScript Console of the Google Chrome web browser application to determine the time of the webpage to fully load. Operating system was the independent variable. Hardware specifications which were classified as old system and new system and types of web content which was also classified as static and dynamic webpages were the intervening variables. Webpages browsing performance was the dependent variable. The statistical tools used were arithmetic mean, and t-test. It also revealed that there were significant differences in the webpage browsing performance between proprietary and open source operating system on wireless networks when classified as to hardware specification and web content. The proprietary and open source operating systems were statistically different when classified as to hardware specifications and type of web content.

KEYWORDS: Open Source Operating Systems, Performance, Proprietary System, Webpage Browsing, Wireless Networks Proprietary

\section{INTRODUCTION}

Not so long ago computers were either large machines housed in carefully controlled rooms accessed via serial terminals, or small home machines which booted from ROM. The personal computer resolutions has brought a computer to each desk, along with the noisy fans disk a modern PC requires, the maintenance nightmare of hundreds of different computer installations to maintain, and the never ending cycle of faster computers being required (McNeill, 2000).

In today's modern technology, network is one of the most valuable comprehensive innovations which pave the way to a much easier and faster data communication. Network is a bridge that fills the gap between two or more computers. Most people correlate network to their day to day kind of living. This innovation uplifts life's standard in such a way that a person can now do many transactions right in front of his computer in the convenience of his own home to the other parts of the world.

The first successful Transatlantic telegraph cable was laid in 1865. A regular Transatlantic radiotelegraph service began in 1907 (www.bizymoms.com).

Packet switching network communication idea seeded in the 1940's, with the conversion of regular telephone networks into digital lines. Advance Research Project Agency (ARPA) started working on a so-called "Inter-galactic" network in 1962, and this was the attempt to establish a sustainable Wide Area Network for communication purposes.

ARPA launched its first successful long distance packet-switching communication network in 1969 with the name ARPanet. It consisted of four universities, which interconnected the researchers working on the project at the time. In the initial communication of ARPAnet, sending only the word "login" to the others end took almost an hour. It is a fact that ARPAnet was the predecessor of the Internet (www.bizymoms.com). 


\section{A Comparative Study on Webpage Browsing Performance between Proprietary and Open Source Operating Systems on Wireless Networks}

ARPA started research in 1970's, looking at means for reducing a network to the bare minimum, while simultaneously joining almost any network. TCP/IP is a layered protocol stack that provides reliability and simplicity in data communications (www.bizymoms.com).

Local Area Network (LAN) communication is another development aside from Internet. Challenging other networking standards like Token-Ring, Ethernet holds the medal to be the standard protocol for LAN communication to its speed and reliability. Ethernet development also began in mid 1970's. Nowadays Ethernet provides staggering 10 gigabits (10GB) per second speeds, known as $10 \mathrm{G}$ Ethernet is still an emerging technology, and remains to receive commercial acceptance (www.bizymoms.com).

By 1973, 75\% of the ARPAnet traffic was email and in 1973, File Transfer Protocol (FTP) was introduced, enabling uploading and downloading of files (www.bizymoms.com).

The version 4 of the TCP/IP was standardized in 1978 with high stability. It is still the underlying standard protocol used even today in the Internet (www.bizymoms.com).

Wireless LANs are continuing to grow in popularity, particularly in enterprise environments that require or desire a high level of mobility. The increased demands for mobility and flexibility in our daily life are demands that lead the development from wired LANs to wireless LANs (WLANs). Today a wired LAN can offer users high bit rates to meet the requirements of bandwidth consuming services like video conferences, streaming video etc. With this in mind a user of a WLAN will have high demands on the system and will not accept too much degradation in performance to achieve mobility and flexibility. This will in turn put high demands on the design of WLANs of the future. A wireless LAN is based on a cellular architecture where the system is subdivided into cells, where each cell (called Base Service Set or BSS) is controlled by a Base station called Access point or AP (Vijay Chandramouli).

In the year 1993, Sir Tim Berners Lee invented the global information highway called the World Wide Web. The emergence of WWW was agreed by the scholars as one of the biggest turning points in computing related network communications in recent history (www.bizymoms.com).

A wireless LAN (WLAN) is analogous to a wired LAN with radio waves being the transport medium instead of traditional wired structures. This allows the users to move around in a limited area while being still connected to the network. Thus, WLANS combine data connectivity with user mobility, and through simplified configuration, enable movable LANs (WLAN Association, 1999). In other words, WLANS provide all the functionality of wired LANs, but without the physical constraints of the wire itself. Generally a WLAN (in Infrastructure mode) consists of a central connection point called the Access Point(AP). It is analogous to a hub or a switch in traditional star topology based wired local area networks. The access point transmits the data between different nodes of a wireless local area network and in most cases serves as the only link between the wireless LAN and the wired LAN.

The wireless nodes, also called clients of a WLAN usually consist of Desktop PCs, Laptops or PDAs equipped with wireless interface cards (WLAN Association, 1999).

Wireless networking is a rapidly changing technology. All users of wireless technology must be aware of the risks inherent in deploying cutting-edge technology. Complete functionality, adherence to standards, and interoperability are all areas in which wireless (or any other new technology) must be closely evaluated to insure a good return on the resources invested in their purchase and use. Wireless messaging is now a dynamic ingredient in the communication modes of our life. Many applications over the Internet now use wireless messages to contact with the end user (Mishra et al., 2004).In this study, the researchers focused on the three factors such as the type of operating system used, hardware specifications and types of web content because they are considered as the common factors that affect the webpage browsing performance on wireless networks. These factors were chosen to determine what type of operating system can deliver a better webpage browsing performance considering the hardware specifications and types of web content.

The operating system, which were classified as proprietary and open source systems, the hardware specification, which were also classified as old (Single Core) and new system (Multi Core), and the type of web content as static and dynamic web pages are three factors that may affect the webpage browsing performance on wireless networks. 
Figure 1 shows the paradigm of the study.

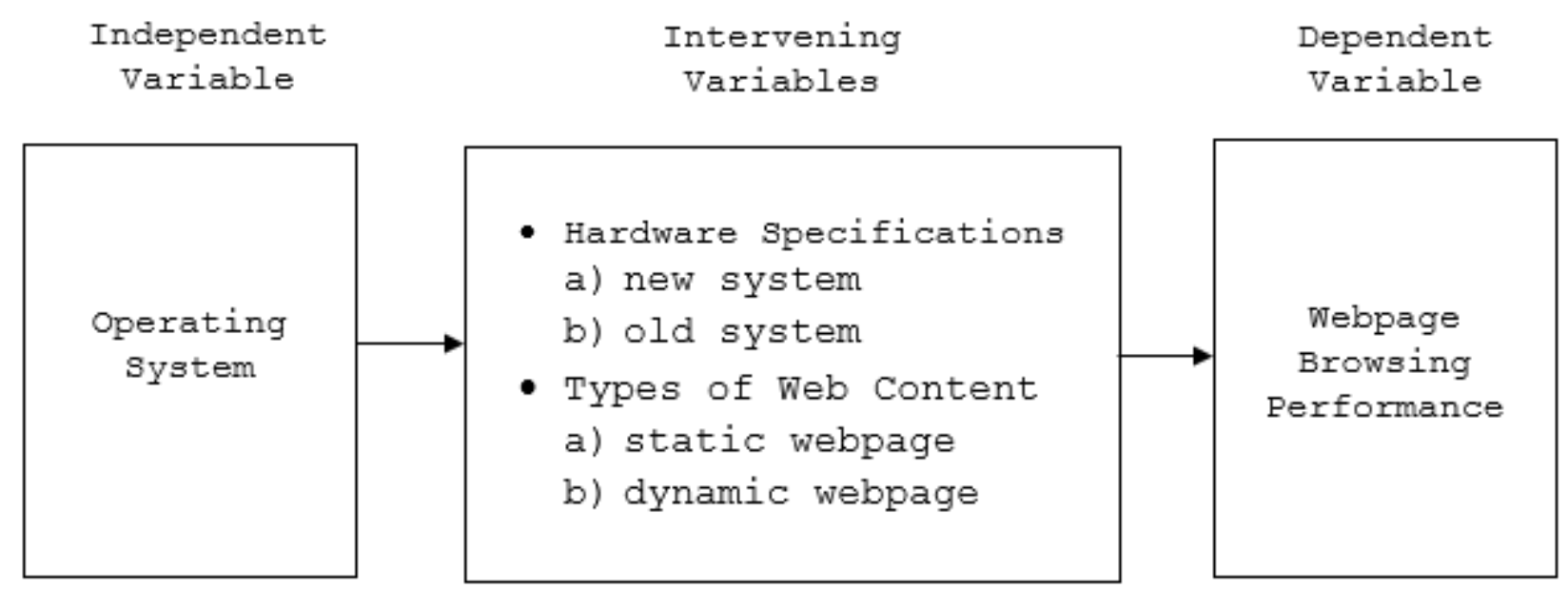

Figure 1. Paradigm of the Study

According to Lucey (2010), processor speed also affects how quick a user can go back and forth between different webpage browsers or tabs. If the user is constantly browsing several different sites simultaneously, the processor speed affects how fast it can load the page. The speed at which the data is delivered to the computer is managed by the speed of the processor.

According to King (2012), since the web page is actually being delivered to the computer, the weight of everything on the page has a major impact on how quickly or slowly the page will load on the end users' screen. In computer jargon, the weight or sizes is measured in bits and bytes. Everything that needs to be transferred including the text used to insert comments into HTML code, has an impact on the overall download time.

This study aimed to identify and compare the webpage browsing performance between proprietary and open source operating systems on wireless networks.

Specifically, this study aimed to answer the following questions:

1. What is the average webpage browsing performance of proprietary operating system on wireless networks when classified according to hardware specifications?

2. What is the average webpage browsing performance of proprietary operating system on wireless networks when classified according to types of web content?

3. What is the average webpage browsing performance of open source operating system on wireless networks when classified according to hardware specifications?

4. What is the average webpage browsing performance of open source operating system on wireless networks when classified according to types of web content?

5. Are there significant differences in the webpage browsing performance between proprietary and open source operating systems on wireless networks when classified as to hardware specifications and types of web content?

The study was only restricted to compare the webpage browsing performance between proprietary and open source operating systems on wireless networks. This study focused on the three factors such as operating system, hardware specifications and types of web content.

There were six fully configured computers that will be used for both proprietary and open source systems on wireless networks.

This was done inside the Bossing Internet Cafe, Cudilla Avenue Street, Estancia, Iloilo. The researchers started the experiment from February 8, 2014 until February 20, 2014.

\section{METHODOLOGY}

\section{Research Design}

This study used an experimental research. According to Key (1997), experimental research is an attempt of the researchers to test the hypothesis by reaching valid conclusions about the relationship between independent and dependent variables.

The researchers observed one element in two different situations to test the hypothesis and come up to a valid conclusion. As the researchers go along with the study, they examined wireless networks using proprietary and open source 


\section{A Comparative Study on Webpage Browsing Performance between Proprietary and Open Source Operating Systems on Wireless Networks}

operating systems and compare the webpage browsing performance of each situation as classified according to hardware specifications and types of web content.

In this study, the researchers focused on three factors namely: operating system, hardware specification and types of web content. The researchers used a wireless clients connected to the web server to access the different web pages stored inside it.

The study used a star topology. In this architecture, the server computer and the six wireless client computers were connected through a wireless router. Each wireless client computers requested web pages on a web server.

\section{Sources of Data}

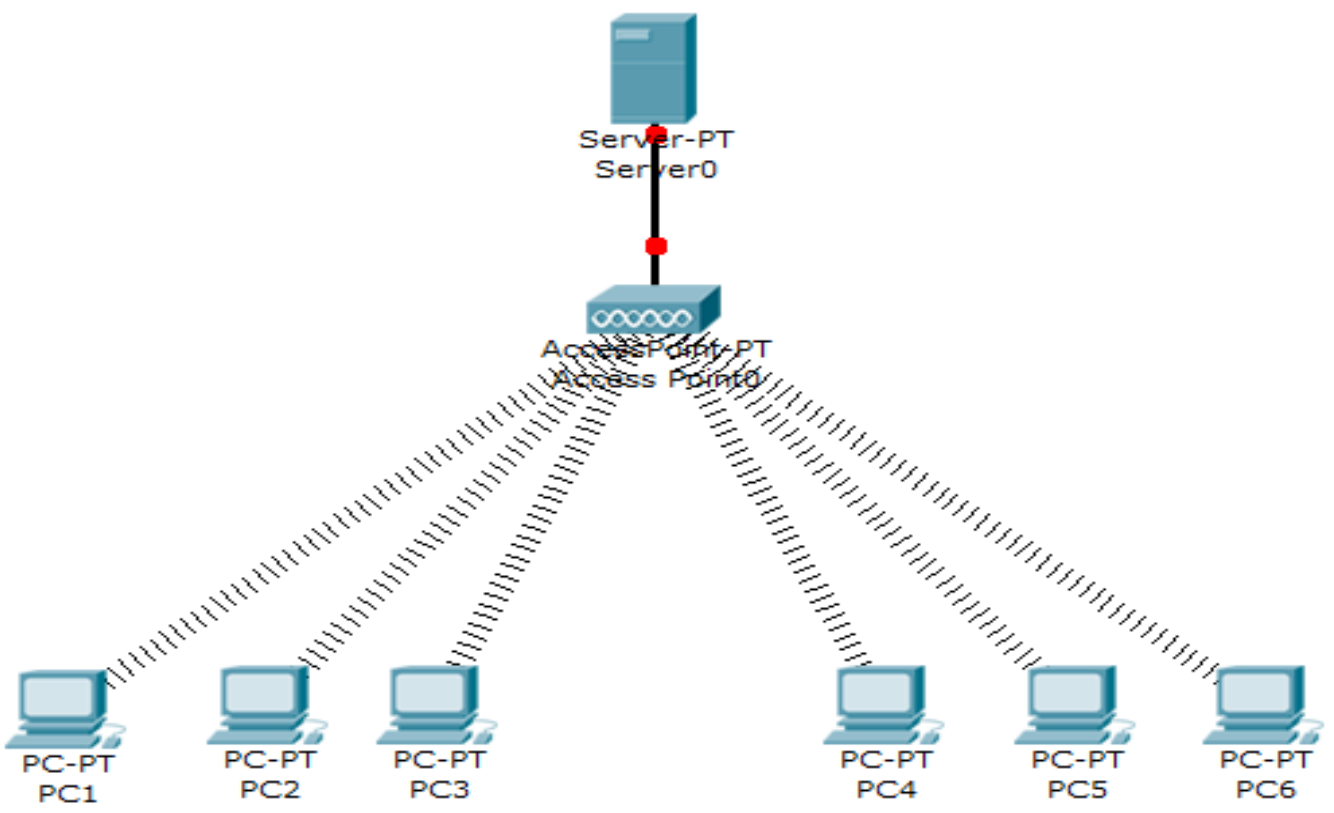

PC4, PC5, and PC6 were categorized under the new system with a Multi Core. PC1, PC2 and PC3 were categorized under the old system with a Single Core.

The computers used in this study were provided with a unique static IP Addresses. The web server was assigned with the IP Addresses of 192.168.100.1. PC1 had an IP Address of 192.168.100.2, 192.168.100.3 for PC2, 192.168.100.4 for PC3, 192.168.100.5 for PC4, 192.168.100.6 for PC5, and 192.168.100.7 for PC6. The above IP Addresses were used on both proprietary and open-source operating system.

In as far as the operating systems are concerned, the researchers decided to use the Windows XP SP3 as the proprietary operating system and the Ubuntu $10.04 \mathrm{DE}$ for the open source operating system. The two operating systems have more or less similar technical specification requirements. The computers that met the requirements were borrowed by the researchers from the Bossing Internet Café.

Table 1 shows the technical specification requirements of Windows XP SP3 and Ubuntu 10.04 DE as the proprietary and open source operating system respectively.

Table 1. Technical Specifications of MS Windows XP and Ubuntu 10.04

\begin{tabular}{|l|l|l|}
\hline Specifications & MS Windows XP & Ubuntu 10.04DE \\
\hline Description & $\begin{array}{l}\text { Can run effectively } \\
\text { in a specified } \\
\text { minimum requirements }\end{array}$ & $\begin{array}{l}\text { Can run effectively } \\
\text { in a specified } \\
\text { minimum requirements }\end{array}$ \\
\hline Processor & 300 megahertz & $300 \mathrm{MHz}$ \\
\hline Memory & 128 megabytes & 128 megabytes \\
\hline Hard DiskSpace & $1.5 \mathrm{~GB}$ & $1 \mathrm{~GB}$ \\
\hline
\end{tabular}

The personal computers used in this study were designated as server and wireless workstations. The server computer was configured to provide operating systems to the clients. Table 4 shows the hardware specification of the computers used in the study. 
A Comparative Study on Webpage Browsing Performance between Proprietary and Open Source Operating Systems on Wireless Networks

Table 2. Hardware Specification of the Computers used in the Study

\begin{tabular}{|c|c|c|c|}
\hline & \multirow[t]{2}{*}{ OS Serve Wireless Computer } & \multicolumn{2}{|c|}{ Client Computers } \\
\hline & & New System & Old System \\
\hline Processor & Intel ${ }^{8} \mathrm{Core}^{\mathrm{TM}} \mathrm{i} 5 \mathrm{CPU}$ & AMD A4-4000APU & Intel ${ }^{\odot}$ Pentium ${ }^{\ominus} 4$ \\
\hline $650 @$ & $320 \mathrm{GHz}$ & $2.99 \mathrm{GHz}$ & $2.80 \mathrm{GHz}$ \\
\hline Memory & $2 \mathrm{~GB}$ & $2 \mathrm{~GB}$ & $2 \times 256 \mathrm{MB}$ \\
\hline Hard Disk & $500 \mathrm{~GB}$ & $500 \mathrm{~GB}$ & $80 \mathrm{~GB}$ \\
\hline
\end{tabular}

\section{Instrumentation and Data Collection}

In the conduct of the study, there were several materials and instruments used that provided the necessary data.

The researchers constructed a data collection form specifically for this study. It was used to reveal the result of the webpage browsing performance. To ensure for its appropriateness, the researchers subjected the instrument to a face validation.

The researchers then looked for the seven computer units including the server in the experiments. Seven computer units were borrowed from Bossing Internet Cafe, and three wireless network interface card were borrowed from Cyberlab Academic Building.

PC1, PC2, PC3 were categorized under the old system and PC4, PC5, PC6 were categorized under the new system with the open source OS and with web content of static. PC1, PC2, PC3 were categorized under the old system and PC4, PC5, PC6 were categorized under the new system with the proprietary OS and with web content of static.

PC1, PC2, PC3 were categorized under the old system and PC4, PC5, PC6 were categorized under the new system with the open source OS and with web content of dynamic. PC1, PC2, PC3 were categorized under the old system and PC4, PC5, PC6 were categorized under the new system with the proprietary OS and with web content of dynamic.

The researchers installed Microsoft Windows Server 2003 Enterprise Edition on the server and Windows XP SP3 on the client computer. The server was also configured to be a DHCP Server to automatically assign IP Addresses to all the clients computers connected to it. The researchers installed Ubuntu 10.04 DE on open source and used it as a server, gave a fixed IP Address and also installed in a client computer.

The researchers were configured the server to be an Apache Web Server by installing Apache 2.2.22 program used for proprietary system on wireless networks. After it was installed, the researchers made some changes on the permissions on an Apache Web Server using a notepad and put inside it the needed static and dynamic web pages to be access by the wireless client computers.

In open source system, the researchers installed Apache 2 and saved the web pages to the Apache folder and used as a local web server by simulating the web content.

After the configuration of proprietary system on wireless networks, the researchers started to test the webpage browsing performance by classifying the type of web content into static and dynamic webpage. There were three static web pages and three dynamic web pages that were stored on the web server. To get a valid result, the researchers tested each web page three times with the use of the JavaScript Console of the Google Chrome web browser. The researchers opened a web browser, then wait until the browser fully load, test it, clear the browsing data, close the browser, the researchers repeat the experiment two times in the same process. For open source system on wireless networks, the same methods on the proprietary system were used to test the web page browsing performance. The gathered data were recorded for interpretation.

The experiment was conducted at the Bossing Internet Cafe at Cudilla Avenue Street, Estancia, Iloilo. The arbitrary scale used in this study was adopted from the scale used by Google Chrome browser when they conducted the average pages load time of top ranking websites in Google.

If the website's average loading time is 9.81 seconds or below, the website is loading "very fast". If the website's average loading time is between 9.82 seconds and 13.84 seconds, then the website URL loading speed is on the "average". If the website's average page loading time falls somewhere 13.85 seconds and 17.98 seconds, the website loading page is "below average". If the website has an average loading time of more than 17.98 seconds, it is considered "very slow". 
A Comparative Study on Webpage Browsing Performance between Proprietary and Open Source Operating Systems on Wireless Networks

Table 3. Showed the summary of the arbitrary scale.

\begin{tabular}{|c|l|}
\hline Range (in seconds) & Description \\
\hline Below 9.82 seconds & Very Fast \\
\hline $9.82-13.84$ & Average \\
\hline $13.85-17.98$ & Below Average \\
\hline 17.99 and above & Very Slow \\
\hline
\end{tabular}

\section{Tools for Data Analysis}

The statistical tools used in the analysis of data are as follows:

Mean. The mean was used to determine the average speed of the webpage browsing performance of proprietary open source operating systems on wireless networks.

T-test. A statistical test that was used to determine if there is significant difference between the mean of two groups in the webpage browsing performance of proprietary and open source operating systems on wireless.

\section{RESULTS AND DISCUSSION}

\section{Descriptive Data Analysis}

The data obtained from the webpage browsing performance of proprietary and open source operating systems on wireless networks when classified according to hardware specification and types of web content.

\section{Average Webpage Browsing Performance of Proprietary \\ Operating System on Wireless Networks when Classified According to Hardware Specifications}

Table 4 shows the average webpage browsing performance of proprietary operating system on wireless networks when classified according to hardware specifications. The gathered data revealed that the obtained mean value for the static 1 webpage was 1.36794 seconds for the old system and was described as "very fast".

On the other hand, the obtained mean value for the static 2 webpage was 0.31449 seconds for the old system and was also described as "very fast". Likewise for the static 3 webpage the mean value was 0.29672 seconds for the old system and was described as "very fast".

The gathered data revealed that the obtained mean value for the dynamic 1 webpage was 5.1100 seconds for the old system and was described as "very fast". In dynamic 2 webpage the mean value was 4.6400 seconds for the old system and also described as "very fast". However, the mean value for the dynamic 3 webpage was 68.6000 seconds for the old system and described as "very slow".

On the new system thegathered data revealed that the obtained mean value was 1.30199 seconds for the static 1 webpage was described as "very fast". At the same time, the obtained mean value for the static 2 webpage was 0.27872 seconds for the new system and was described as "very fast". For the static 3 webpage the mean value was 0.20811 seconds for the new system and was also described as "very fast".

The gathered data obtained that the dynamic 1 webpage was 4.7767 seconds for the new system and was described as "very fast". For the dynamic 2 webpage the obtained mean value 5.4333 seconds for the new system and was described as "very fast". In the new system the obtained mean value was 69.8000 seconds for the dynamic 3 webpage and described as "very slow". 
A Comparative Study on Webpage Browsing Performance between Proprietary and Open Source Operating Systems on Wireless Networks

Table 4. Average Webpage Browsing Performance of ProprietaryOperating System on Wireless Network whenClassified According to HardwareSpecifications

\begin{tabular}{lrll}
\hline $\begin{array}{l}\text { Types of } \\
\text { Web ContentHardware }\end{array}$ & Types of & Mean & Description \\
Specification & & & \\
\hline (sec) & & \\
Static 1 & & & Very Fast \\
Static 2 & Old System & 1.36794 & Very Fast \\
Static 3 & Old System & 0.31449 & Very Fast \\
Dynamic 1 & Old System & 0.29672 & Very Fast \\
Dynamic 2 & Old System & 5.1100 & Very Fast \\
Dynamic 3 & Old System & 4.6400 & Very Slow \\
\hline Static 1 & Old System & 68.6000 & Very Fast \\
Static 2 & New System & 1.30199 & Very Fast \\
Static 3 & New System & 0.27872 & Very Fast \\
Dynamic 1 & New System & 0.20811 & Very Fast \\
Dynamic 2 & New System & 4.7767 & Very Fast \\
Dynamic 3 & New System & 5.4333 & Very Slow \\
\hline
\end{tabular}

$\begin{array}{cl}\text { Scale } & \text { Description } \\ \text { Below } 9.82 \text { seconds } & \text { Very Fast } \\ 9.83-13.84 \text { seconds } & \text { Average } \\ 13.85-17.98 \text { seconds } & \text { Below Average } \\ 17.90 \text { seconds and Above } & \text { Very Slow }\end{array}$

\section{Average Webpage Performance of Proprietary and Open Source Operating System on Wireless Network when Classified \\ According to Types of Web Content}

Table 5 shows the average webpage browsing performance or proprietary and open source operating system on wireless networks when classified according to types of web content. The gathered data revealed that the obtained mean value for the static 1 webpage was 0.23661 seconds for the open source operating system was described as "very fast". On the other hand, the obtained mean value for the static 2 webpage was 0.22778 seconds for the open source operating system and was also described as "very fast". For the static 3 webpage the mean value was 0.27022 seconds for the open source operating system which described as "very fast".

The mean value for static 2 webpage was 0.22778 seconds and was described as "very fast". On the dynamic 2 webpage the mean value was 2.57830 seconds and was also described "very fast".

The gathered data revealed for the obtained mean value for static 3 webpage was 0.27022 seconds which was described as "very fast. For the dynamic 3 webpage the mean value was 69.00000 seconds and was described as "very slow".

For the dynamic 1 webpage for the open source operating system the mean value was 1.90000 seconds which was described as "very fast". On the open source operating system for the dynamic 2 webpage the mean value was 2.57830 seconds which was described as "very fast". For the dynamic 3 webpage for the open source operating system the mean value was 69.00000 seconds and was described as "very slow".

The gathered data obtained for the proprietary operating system for the static 1 webpage was 2.43333 seconds which was described as "very fast". For the static 2 webpage the mean value was 0.36544 seconds for the proprietary operating system which was also described as "very fast". Likewise, on the static 3 webpage the mean value was 0.23461 seconds for the proprietary operating system and was described as "very fast".

The obtained mean value for the dynamic 1 webpage for the proprietary operating system was 7.98670 seconds which was described as "very fast". For the dynamic 2 webpage the mean value was 7.50000 seconds for the proprietary operating 
A Comparative Study on Webpage Browsing Performance between Proprietary and Open Source Operating Systems on Wireless Networks

system which was also described as "very fast". However, for the dynamic 3 webpage the mean value was 69.40000 seconds for the proprietary operating system and described as "very slow".

Table 5. Average Webpage Browsing Performance of Proprietary and Open Source Operating System on Wireless Network When Classified According to Types of Web content

\begin{tabular}{|c|c|c|c|}
\hline $\begin{array}{l}\text { Type of Web } \\
\text { Content }\end{array}$ & \multicolumn{2}{|c|}{$s(\sec )$} & Description \\
\hline Static 1 Webpage & Open Source & 0.23661 & Very Fast \\
\hline Static 2 Webpage & Open Source & 0.22778 & Very Fast \\
\hline Static 3 Webpage & Open Source & 0.27022 & Very Fast \\
\hline Dynamic 1 Webpage & Open Source & 1.90000 & Very Fast \\
\hline Dynamic 2 Webpage & Open Source & 2.57830 & Very Fast \\
\hline Dynamic 3 Webpage & Open Source & 69.00000 & Very Slow \\
\hline Static 1 Webpage & Proprietary & 2.43333 & Very Fast \\
\hline Static 2 Webpage & Proprietary & 0.36544 & Very Fast \\
\hline Static 3 Webpage & Proprietary & 0.23461 & Very Fast \\
\hline Dynamic 1 Webpage & Proprietary & 7.98670 & Very Fast \\
\hline Dynamic 2 Webpage & Proprietary & 7.50000 & Very Fast \\
\hline Dynamic 3 Webpage & Proprietary & 69.4000 & Very Slow \\
\hline \multicolumn{2}{|c|}{ Scale } & \multicolumn{2}{|c|}{ Description } \\
\hline \multicolumn{2}{|c|}{ Below 9.82 seconds } & \multicolumn{2}{|c|}{ Very Fast } \\
\hline \multicolumn{2}{|c|}{$9.83-13.84$ seconds } & \multicolumn{2}{|c|}{ Average } \\
\hline \multicolumn{2}{|c|}{$13.85-17.98$ seconds } & \multicolumn{2}{|c|}{ Below Average } \\
\hline \multicolumn{2}{|c|}{17.90 seconds and Above } & \multicolumn{2}{|c|}{ Very Slow } \\
\hline
\end{tabular}

Inferential Data Analysis

After the conduct of the study, the results were statistically computed. The Mean and the T-test were used as the inferential statistical tool.

Difference in the Webpage Browsing Performance between

Proprietary and Open Source Operating Systems on Wireless

Networks using Different Hardware Specification

The table 6 shows the webpage browsing performance between proprietary and open source operating systems on wireless networks when classified according to hardware specification. The obtained Sig. (2-tailed) value was 0.000 for dynamic 1 and dynamic 2 webpages, 0 . Hence, the alternative hypothesis states that there are significant differences in the webpage browsing performance between proprietary and open source operating systems using different hardware specification that were accepted. Table 6. Difference in the Webpage Browsing Performance between Proprietary and Open Source Operating Systems when Classified According to Hardware Specification

\begin{tabular}{|c|c|c|c|}
\hline \multicolumn{4}{|l|}{ Types of Web } \\
\hline Contez t & df & \multicolumn{2}{|c|}{ Sige(2-tailed) } \\
\hline Static 1 Webpage & 0.095 & \multicolumn{2}{|c|}{$10 \quad 0.926$} \\
\hline Static 2 Webpage & 0.61010 & \multicolumn{2}{|c|}{0.556} \\
\hline Static 3 Webpage & 0.972 & \multicolumn{2}{|c|}{$10 \quad 0.354$} \\
\hline Dynamic 1 Webpage & -16.789 & 10 & $0.000^{*}$ \\
\hline Dynamic 2 Webpage & -9.670 & 5.189 & $9 \quad 0.000^{*}$ \\
\hline Dynamic 3 Webpage & -0.542 & 10 & 0.599 \\
\hline
\end{tabular}

*significant at 0.05 alpha level. 


\section{A Comparative Study on Webpage Browsing Performance between Proprietary and Open Source Operating Systems}

on Wireless Networks

\section{Difference in the Webpage Browsing Performance between \\ Proprietary and Open Source Operating Systems on Wireless \\ Networks using Different Web Content}

The table 7 shows the webpage browsing performance between proprietary and open source operating systems on wireless networks when classified according to web content specifications. The obtained Significant (2-tailed) value was 0.000 for static 1 and static 2 webpage, 0.000 for the dynamic 1 and dynamic 2 webpages. Hence, the alternative hypothesis states that there are significant differences in the webpage browsing performance between proprietary and open source operating systems using different web content that were accepted.

Table 7. Difference in the Webpage Browsing Performance between Proprietary and Open Source Operating Systems when Classified According to Web Content

\begin{tabular}{|c|c|c|c|}
\hline $\begin{array}{l}\text { Types of Web } \\
\text { Content }\end{array}$ & $\mathrm{t}$ & $\mathrm{df}$ & Sig.(2-tailed) \\
\hline Static 1 Webpage & -58.352 & 10 & $0.000^{*}$ \\
\hline Static 2 Webpage & -3.363 & 10 & $0.000^{*}$ \\
\hline Static 3 Webpage & 0.972 & 10 & 0.354 \\
\hline Dynamic 1 Webpage & -16.789 & 10 & $0.000^{*}$ \\
\hline Dynamic 2 Webpage & -9.670 & \multicolumn{2}{|c|}{$5.1890 .000^{*}$} \\
\hline Dynamic 3 Webpage & -0.542 & 10 & 0.599 \\
\hline
\end{tabular}

*significant at 0.05 alpha level.

\section{CONCLUSION}

The main purpose of this study was to determine and compare the webpage browsing performance between proprietary and open source operating systems on wireless networks.

Specifically, this study sought to answers to the following questions:

1. What is the average webpage browsing performance of proprietary operating system on wireless networks when classified according to hardware specifications?

2. What is the average webpage browsing performance of proprietary operating system on wireless networks when classified according to types of web content?

3. What is the average webpage browsing performance of open source operating system on wireless networks when classified according to hardware specifications?

4. What is the average webpage browsing performance of open source operating system on wireless networks when classified as to hardware specifications?

5. Are there significant differences in the webpage browsing systems on wireless networks when classified as to hardware specifications and types of web content?

Experimental research was used in the study. The researchers used seven computers, router, JavaScript Console of Google Chrome, Microsoft Server Enterprise Edition 2003 was used as the server, for proprietary system Microsoft Windows XP SP3 was used for wireless proprietary operating system. Ubuntu 10.04 Desktop Edition was used for wireless clients in open operating system. The computers were configured to be a wireless client computers. The researcher installed Apache Web Server on the server computer.

Permission to borrowed three wireless network interface card was secured by the researchers from the director of the Institute of Information and Computer Studies and the chairperson of the BSAIT program. The data gathered for the study were subjected to statistical treatment utilizing the Arithmetic Mean and T-test.

The average webpage browsing performance of proprietary operating system on wireless networks when classified according to hardware specifications and types of web content regarded the result as very fast. Similarly, the average webpage browsing performance of open source operating system on wireless network when classified according to hardware specifications and type of web content was also described as very fast. 


\section{A Comparative Study on Webpage Browsing Performance between Proprietary and Open Source Operating Systems on Wireless Networks}

Based on the analysis of the findings of the study, the researchers concluded that the webpage loading time of open source operating system and proprietary operating system revealed a the same performance considering the hardware specifications and types of content. Both operating systems were interpreted as very fast, it was still revealed that open source operating system loads faster.

Similarly, there was a significant difference in the webpage browsing performance between proprietary and open source operating system on wireless networks when classified as to hardware specifications and types of web content. This means that the webpage loading time on wireless networks when classified according to hardware specification and type of web content delivers a different performance between proprietary and open source operating system.

In view of the findings in this study, the researchers recommend that different operating systems and web browsers should be used for similar study having the same factors. The same operating system but different web browser and factors should be used.

\section{REFERENCES}

\section{Books}

1) PARSONS, J. J and OJA, D. 2000. New Perspectives on

2) Computer Concepts. 4th ed. Course Technology,

3) Thomson Learning.

4) TANENBAUM, A. 2003. Computers Networks. $4^{\text {th }}$ ed. UpperSanddle River, NJ: Prentice Hall PTR.

5) WELLS, D. 2009. Basic Computer Concepts. PhilippineEdition,5 Shenton Way \#01-01 UIC Building Singapore:engage Learning.

\section{Electronic Resource}

1) Average Page Load Time of Top Ranking Websites in Google.Retrieved from http://www.seochat.com/c/a/googleOptimization-help/averge-page-load-time-of-top-Ranking-websites-in-google/; Retrieved on: January 18,2013

2) Evolution of Networking. Retrieved fromhttp://www.bizymoms.com/computers-andtechnology/network-evolution.html; November 21, 2012

3) http://www.answers.com/topic/proprietary-perating-system;Retrieved on January 11, 2013

4) http://www.wright edu.com/definition/wireless workstation; Retrieved on February 22, 2012

5) http://www.wifinotes.com/computer-hardware-

6) components/computer-hardware-specification.html;Retrieved on January 10, 2013

7) http://www.wisegeek.com/defintion/dynamic-and-static; Retrieved on January 22, 2012

8) Like New Computers Definition. Retrieved fromwww.likenewcomputers.com/status.php; Retrieved onJanuary 21, 2013 